\title{
Windshear Identification Algorithms by Doppler Pulse Lidar
}

\author{
Nikolay Baranov ${ }^{1, *}$, Ekaterina Lemishchenko ${ }^{2}$ \\ ${ }^{1}$ Dorodnicyn Computing Centre, Federal Research Center “Computer Science and Control” of Russian Academy of Sciences, Moscow, \\ Russia \\ ${ }^{2}$ International Aero Navigation Systems Concern, JSC. Moscow, Russia
}

\begin{abstract}
Doppler pulse lidars are increasingly common as a tool for monitoring hazardous wind phenomena. Possible applications include measurements of wake vortices behind aircraft, detection of vertical and horizontal wind shear, detection of gust fronts, and identification of areas of intense turbulence. This paper reviews several computational procedures used to measure wind shear parameters and determine the gust front position. Algorithms for wind shear calculation are based on the construction of regression dependencies of changes in the wind speed components at a given distance. In accordance with the ICAO recommendations, horizontal wind shear is determined at a distance of $600 \mathrm{~m}$. The application of the algorithms is illustrated by the results of data processing for scanning in PPI mode performed by WINDEX5000 Doppler pulse lidar.
\end{abstract}

\section{Introduction}

Wind shear is one of the most challenging meteorological phenomena complicating aviation operations. This phenomenon is especially dangerous at low altitudes and can lead to aviation incidents and even aviation accidents. In order to improve flight safety, it is vital is to ensure continuous monitoring of wind conditions in the near-ground layer of the atmosphere. Doppler lidars have been increasingly utilized for the purpose of measuring radial components of the wind speed over the past decade.

The advantage of using these tools for measuring wind parameters is determined by the possibility of identifying wind situation characteristics at extremely low altitudes and distances of $1-5 \mathrm{~km}$ from the runway threshold. A significant number of publications [1-7] are devoted to the use of ground-based lidars for wind shear identification. However, as a rule, the issues of algorithmic processing of lidar measurements for identification remain outside the scope.

This paper reviews an algorithm for determining wind shear parameters based on the least squares method with weights of regression dependencies for measured or reconstructed wind speed components. The work is based on the classical criterion for determining wind shear as the magnitude of the change in wind speed [8, 9] at a given distance and does not cover the issues of determining such a wind shear indicator as the Ffactor [10-12].

\section{Windshear Identification Algorithm}

Let $w$ be an arbitrary component of the wind speed $\left(u_{x}\right.$, $\left.u_{z}, u_{r}, u\right)$. The components considered are the Cartesian

\footnotetext{
* Corresponding author: baranov@ians.aero
}

components of the horizontal wind velocity $u_{x}, u_{z}$, the radial wind velocity component $u_{r}$, and the absolute value of the horizontal wind velocity $u$.

The wind shear at the point $\boldsymbol{r}_{0}$ in the direction given by the guiding vector $\boldsymbol{e}$ is determined as the change of this component at the distance $L_{W S}$ :

$$
W S_{w}=w\left(\boldsymbol{r}_{0}+0.5 L_{W S} \boldsymbol{e}\right)-w\left(\boldsymbol{r}_{0}-0.5 L_{W S} \boldsymbol{e}\right) .
$$

To reduce the effect of random fluctuations in the wind speed due to the atmosphere turbulence, as well as measurement errors, the characteristics of the wind field at the considered distance are smoothed.

To determine the wind shear at the point $\boldsymbol{r}_{0}$ in the direction given by the guiding vector $\boldsymbol{e}$, the components of the wind speed $\left(u_{x j}, u_{z j}\right)$ are calculated at the points with coordinates

$$
\boldsymbol{r}_{j}=\boldsymbol{r}_{0}+(j-0.5 N) \boldsymbol{e} / N .
$$

The headwind/crosswind component and the absolute value of the wind speed are calculated.

$$
\begin{gathered}
u_{r j}=e_{x} u_{x j}+e_{z} u_{z j}, \\
u_{j}^{2}=u_{x j}{ }^{2}+u_{z j}{ }^{2},
\end{gathered}
$$

$e_{x}, e_{z}$ are the components of vector $\boldsymbol{e}$.

A linear approximation is constructed using the weighted least squares method for each wind speed component

$$
w\left(\boldsymbol{r}_{j}+0.5 L_{W S} \boldsymbol{e}\right) \approx a_{w}+b_{w}\left(\boldsymbol{r}_{j}-\boldsymbol{r}_{0}\right) .
$$

With such an approximation, the parameter $b_{w}$ in this case is the gradient of the wind speed component $w$ at the distance 


$$
\left[\boldsymbol{r}_{0}-0.5 L_{W S} \boldsymbol{e}, \boldsymbol{r}_{0}+0.5 L_{W S} \boldsymbol{e}\right],
$$

while the value $b_{w} \cdot L_{W S}$ determines the magnitude of the wind shear, calculated based on the change in the wind speed component $w$.

Initially the first approximation is constructed using the least squares method without weights:

$$
\min \Sigma_{j}\left\{w\left(\boldsymbol{r}_{j}+0.5 L_{W S} \boldsymbol{e}\right)-a_{w 1}-b_{w 1}\left|\boldsymbol{r}_{j}-\boldsymbol{r}_{0}\right|\right\}^{2} .
$$

The coefficients $a_{w 1}, b_{w 1}$ are calculated as a solution to the set of equations

$$
M \cdot C=Z,
$$

where

$$
\begin{gathered}
M_{11}=1, M_{12}=M_{21}=\left(\Sigma_{j} l_{j}\right) / N, M_{22}=\left(\Sigma_{j} l_{j}^{2}\right) / N, \\
C_{1}=a_{w 1}, C_{2}=b_{w 1}, \\
Z_{1}=\left(\Sigma_{j} w_{j}\right) / N, Z_{2}=\left(\Sigma_{j} w_{j} l_{j}\right) / N, \\
w_{j}=w\left(\boldsymbol{r}_{j}+0.5 L_{W S} \boldsymbol{e}\right), \\
l_{j}=\left|\boldsymbol{r}_{j}-\boldsymbol{r}_{0}\right|=(j-0.5 N) L_{W S} / N .
\end{gathered}
$$

Approximation errors are calculated

$$
\delta_{j}^{2}=\left\{w\left(\boldsymbol{r}_{j}+0.5 L_{W S} \boldsymbol{e}\right)-a_{w 1}-b_{w 1}\left|\boldsymbol{r}_{j}-\boldsymbol{r}_{0}\right|\right\}^{2} .
$$

After that, the final values of the coefficients $a_{w}, b_{w}$ are calculated as a solution to the set of equations

$$
\boldsymbol{M}_{\delta} \cdot \boldsymbol{C}=\mathbf{Z}_{\delta},
$$

where

$$
\begin{gathered}
M_{\delta 11}=\left\{\Sigma_{j}\left(1 / \delta_{j}^{2}\right)\right\} / N, M_{\delta 12}=M_{\delta 21}=\left\{\Sigma_{j}\left(l_{j} / \delta_{j}^{2}\right)\right\} / N, \\
M_{\delta 22}==\left\{\Sigma_{j}\left(l_{j}^{2} / \delta_{j}^{2}\right)\right\} / N, \\
Z_{\delta 1}=\left\{\Sigma_{j}\left(w_{j} / \delta_{j}^{2}\right)\right\} / N, Z_{\delta 2}==\left\{\Sigma_{j}\left(w_{j} l_{j} / \delta_{j}^{2}\right)\right\} / N,
\end{gathered}
$$

which is obtained from the condition of the weighted approximation error minimum

$$
\min \Sigma_{j}\left\{w\left(\boldsymbol{r}_{j}+0.5 L_{W S} \boldsymbol{e}\right)-a_{w 1}{ }^{-} b_{w 1}\left|\boldsymbol{r}_{j}-\boldsymbol{r}_{0}\right|\right\}^{2} / \delta_{j}^{2} .
$$

After the approximation coefficients are calculated for each wind speed component, the wind shear values are calculated for each criterion:

- wind shear on the magnitude of change in the headwind/crosswind component

$$
W S_{r}\left(\boldsymbol{r}_{0}\right)=b_{w}\left(\boldsymbol{r}_{0}\right) L_{W S},
$$

where $b_{w}\left(\boldsymbol{r}_{0}\right)$ is the coefficient of approximation of the headwind/crosswind component $u_{r}$ of the wind speed at the distance $\left[\boldsymbol{r}_{0}-0.5 L_{W S} \boldsymbol{e}, \boldsymbol{r}_{0}+0.5 L_{W S} \boldsymbol{e}\right]$;

- wind shear on the magnitude of change in the absolute wind speed

$$
W S_{u}\left(\boldsymbol{r}_{0}\right)=b_{u}\left(\boldsymbol{r}_{0}\right) L_{W S},
$$

where $b_{u}\left(\boldsymbol{r}_{0}\right)$ is the coefficient of approximation of the absolute wind speed $u$ at the distance $\left[\boldsymbol{r}_{0}-0.5 L_{W S} \boldsymbol{e}\right.$, $\left.\boldsymbol{r}_{0}+0.5 L_{W S} \boldsymbol{e}\right]$

- wind shear on the magnitude of change in the wind speed and direction

$$
W S_{\text {grad }}\left(\boldsymbol{r}_{0}\right)=L_{W S} \downarrow\left\{b_{x}\left(\boldsymbol{r}_{0}\right)^{2}+b_{z}\left(\boldsymbol{r}_{0}\right)^{2}\right\},
$$

where $b_{x}\left(\boldsymbol{r}_{0}\right)^{\prime} b_{z}\left(\boldsymbol{r}_{0}\right)$ are the coefficients of approximations of Cartesian wind velocity components $u_{x}, u_{z}$ correspondingly at the distance $\left[\boldsymbol{r}_{0}-0.5 L_{W S} \boldsymbol{e}, \boldsymbol{r}_{0}+0.5 L_{W S}\right.$ $e]$.

The point $\boldsymbol{r}_{0}$ determines the spatial reference of the calculated wind shear.

\section{Gust Front Detection Algorithm}

To determine the position of the gust front, a distance section of the length $L_{W G}$ is considered.

The gust front is understood as an intensive change in the headwind/crosswind component of the wind speed with a change in sign. The position of the gust front is identified as the change point of the sign of the approximation velocity dependence.

To determine the position of the gust front, the measured values of the radial component of the wind speed are approximated by a logistic type function:

$$
f(r)=1 /(1+\exp (a+b \cdot r)),
$$

where $a, b$ are the approximation coefficients, and $r$ is the range parameter. To build a logistic approximation, the measured values of the radial wind speed component are converted to

$$
q_{j}=\ln \left\{\left(V_{\max }-V_{\min }\right) /\left(u_{r j}-V_{\min }\right)-1\right\},
$$

where

$$
\begin{gathered}
V_{\min }=\min \left\{u_{r j}\right\}-\varepsilon, \\
V_{\max }=\max \left\{u_{r j}\right\}^{-} \varepsilon .
\end{gathered}
$$

Minimized function will be

$$
\Sigma_{j}\left(w_{j}-a-b \cdot r_{j}\right)^{2} .
$$

The approximation coefficients are calculated as a solution to a set of equations similar to the set of equations (1):

After the logistic approximation coefficients are calculated, the front position is calculated by the formula $r_{W G}=\left(q_{\text {lim }}-a\right) / b$,

where

$$
q_{\text {lim }}=\ln \left\{-\left(V_{\max }-V_{\min }\right) / V_{\min }-1\right\} .
$$

To illustrate the application of this approach, Figure 1 shows a fragment of data from lidar measurements in LoS mode of the radial wind speed projection performed by a Doppler pulse lidar over a section $1 \mathrm{~km}$ long and the results of constructing an exponential approximation of experimental data. The dots show the measured velocity values, the dotted line represents the calculated smoothed approximation. The identified gust front 
position is shown by a blue vertical line.

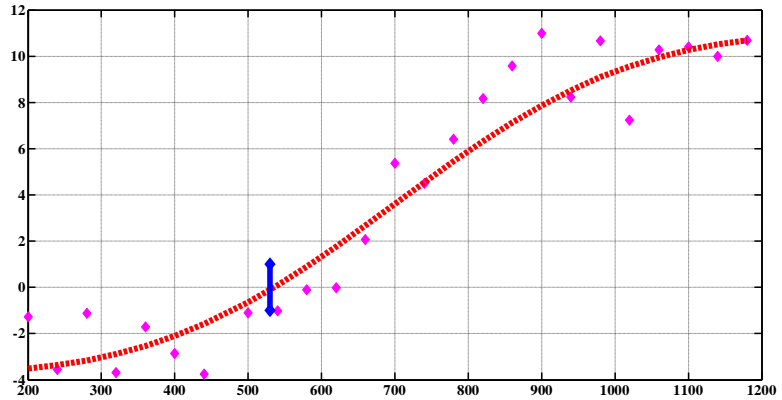

Fig. 1. Example of constructing a smoothing exponential function of lidar measurement data

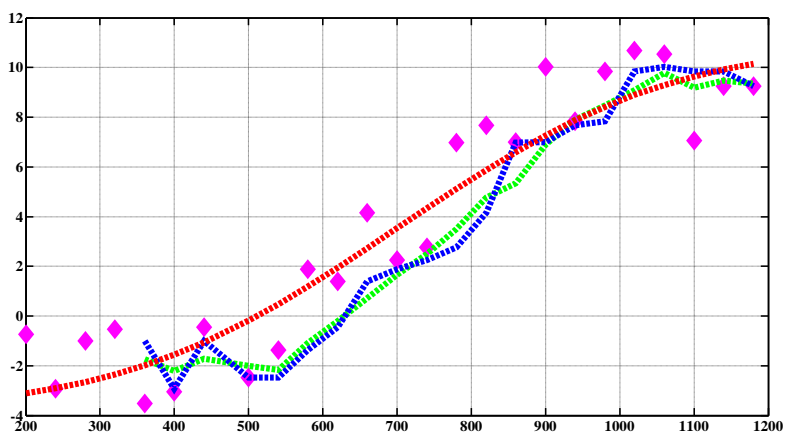

Fig. 2. Comparison of exponential, average, and median smoothing

\section{Unreliable Data Filtering}

The first step in the procedure for determining wind shear parameters is filtering the lidar measurement data by the threshold level of the signal-to-noise ratio. Only reliable measurement data that have been pre-filtered are used in further processing.

Likewise, additional filtering conditions for outliers are used:

1. A measurement point at which the SNR value exceeds a specified threshold value must have at least one adjacent measurement point at which the SNR also exceeds the threshold value, otherwise this point is considered an outlier and its value is not taken into account in further calculations;

2. As a result of data selection for calculating the wind phenomenon parameters (wind shear or gust front position), a set of reliable data for the calculation should be sufficiently representative. The condition for the representativeness of the selected measurement data is the excess in number of selected measurement points of the specified minimum value.

3. The selected measurement points should, in a sense, fill almost the entire interval examined for wind shear. Mathematically, this means that

$$
\max \left\{\left(\boldsymbol{r}_{j}, \boldsymbol{e}\right)\right\}-\min \left\{\left(\boldsymbol{r}_{j}, \boldsymbol{e}\right)\right\} \approx L_{W S} .
$$

In addition to the filtering procedures for unreliable data, the arithmetic mean algorithm or the average smoothing algorithm are used which on the one hand reduce random fluctuations of the measured values and on the other act as a procedure for restoring missing measurements. As a rule, three- or five-point averaging is used.

\section{Result and discussion}

First, let us illustrate the operation of smoothing algorithms in adverse measurement conditions. The results of measurements obtained using WINDEX-5000 Doppler pulse lidar jointly developed by International Aero Navigation Systems Concern, JSC and Laser Systems LLC were used as initial data.

Figure 3 shows the results of measurements in PPI (Plan Position Indicator) mode of the wind field in poor visibility conditions (meteorological visibility less than $1000 \mathrm{~m})$. PPI mode corresponds to measurements at a constant elevation angle and different azimuth angles of the measurement direction. Thus, it is obvious that if the elevation angle of the scanning of the Doppler lidar is non-zero, the measurement data take into account the change in the wind field not only by range but also by height. Therefore, lidar scanning data in PPI mode generally takes into account not only horizontal wind shear (wind shear in the horizontal plane), but also vertical wind shear (changes in wind speed and directionby heigh).

Scanning was performed with an elevation angle of $5^{\circ}$. Missing data in the azimuth angle range of $90^{\circ}-180^{\circ}$ is due to the presence of low-height small obstacles in the scanning area.

For comparison, Figures 4 and 5 show the results of processing wind scan data after applying various types of smoothing algorithms. It can be seen that the use of smoothing algorithms ensures completion of the missing measurement data. At the same time, the quality of recovering the missing data is about the same for the two types of algorithms.

Figures 6 and 7 show, respectively, the field of measured radial velocities and the wind shear field calculated from the magnitude of the change in the radial component. Scanning was performed with an elevation angle of $13^{\circ}$. The pattern of radial velocities distribution shows the presence of an intense change in the projections of radial velocity in the south-west-northeast direction in the range of $2000-3000 \mathrm{~m}$. This change is due to the typical dangerous wind phenomenon of low-level vertical wind shear by speed which is observed in the height range of 500-700m. The wind shear distribution corresponding to such a wind shear has a characteristic appearance with concentric areas of positive and negative wind shear corresponding to the ranges in which the increase and decrease of the radial velocity are observed.

Another typical dangerous wind shear phenomenon is shown in Figure 9. The distribution of radial velocities has a characteristic spiral pattern which corresponds to a wind shear by direction [13]. The corresponding distribution of parameters of the wind shear by the radial velocity has a similar structure (Fig. 10). 


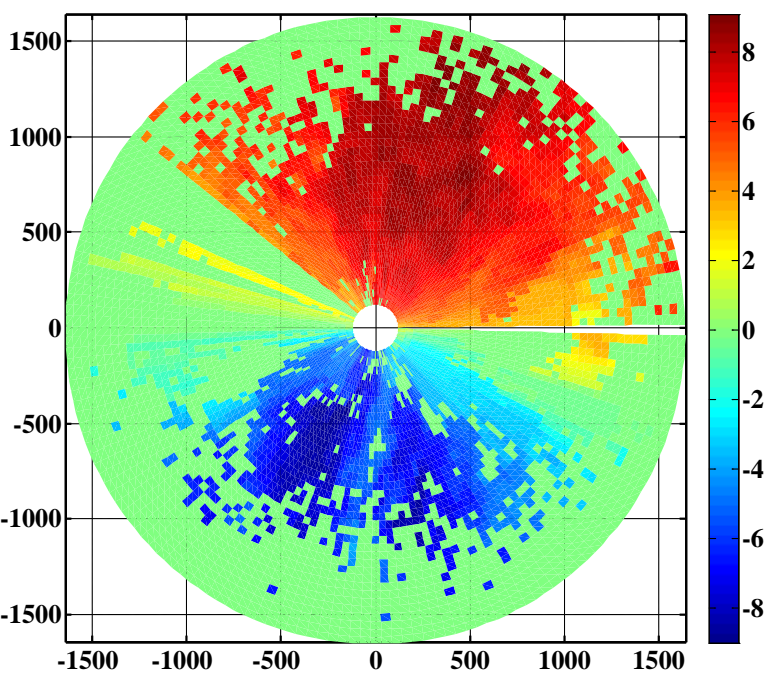

Fig. 3. Velocity field measured in PPI mode in poor visibility conditions

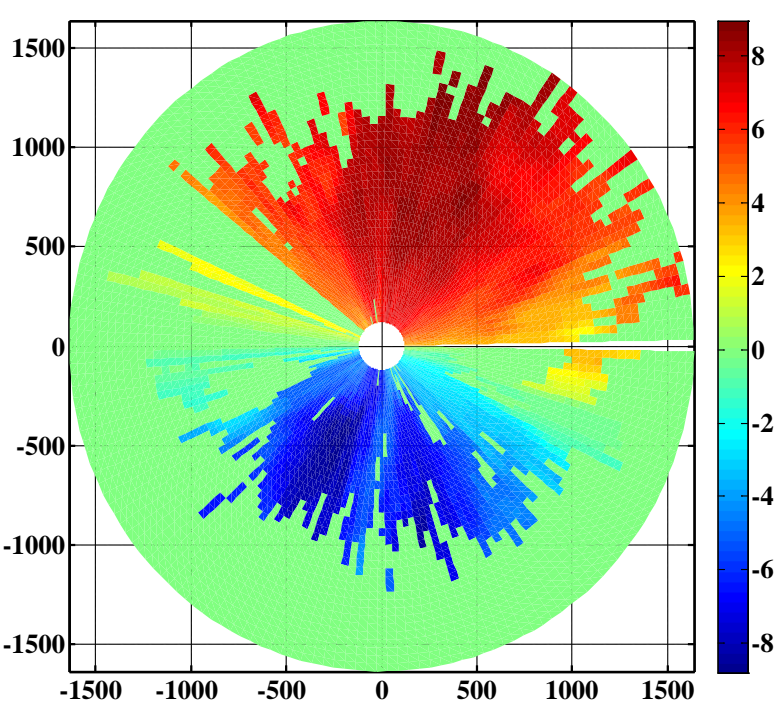

Fig. 4. Distribution of radial velocities after the application of the arithmetic mean smoothing algorithm

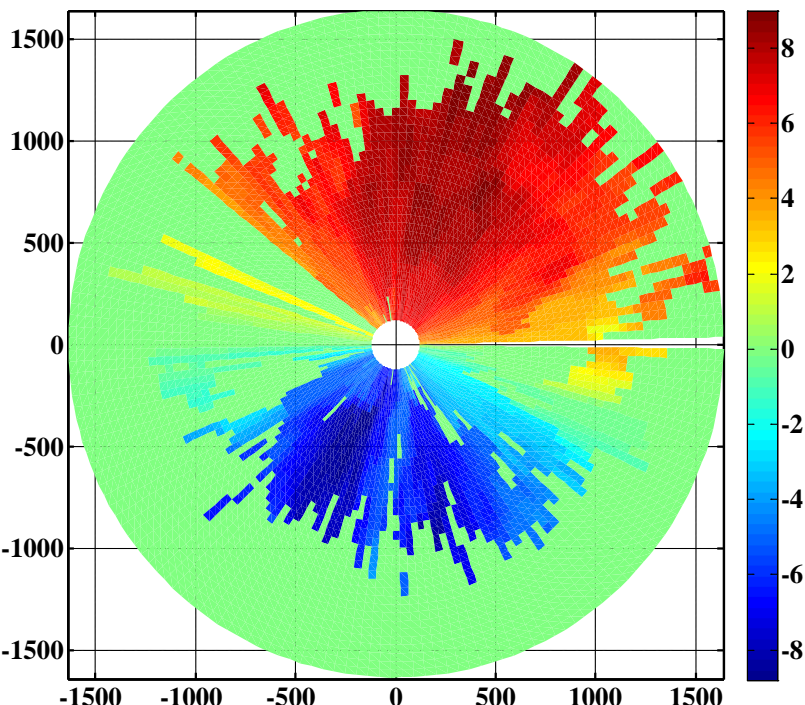

Fig. 5. Radial velocity distribution after applying the median smoothing algorithm

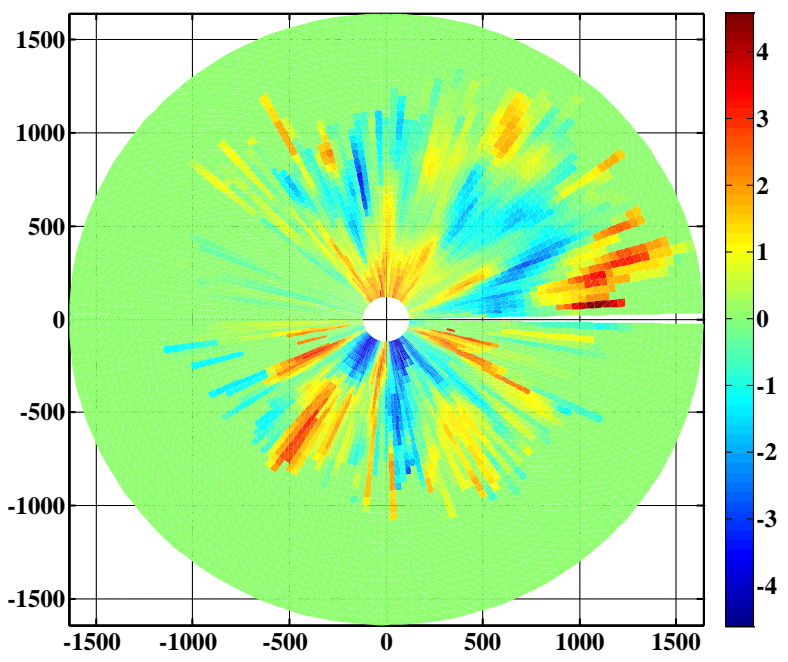

Fig. 6. Radial velocity shear field obtained after postprocessing of measurement data in poor visibility conditions

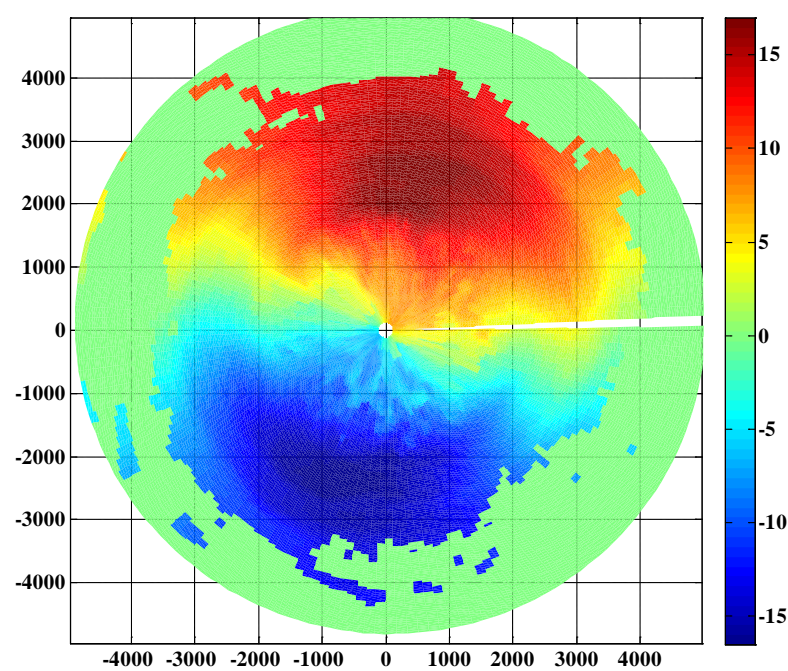

Fig. 7. Velocity field measured in PPI mode

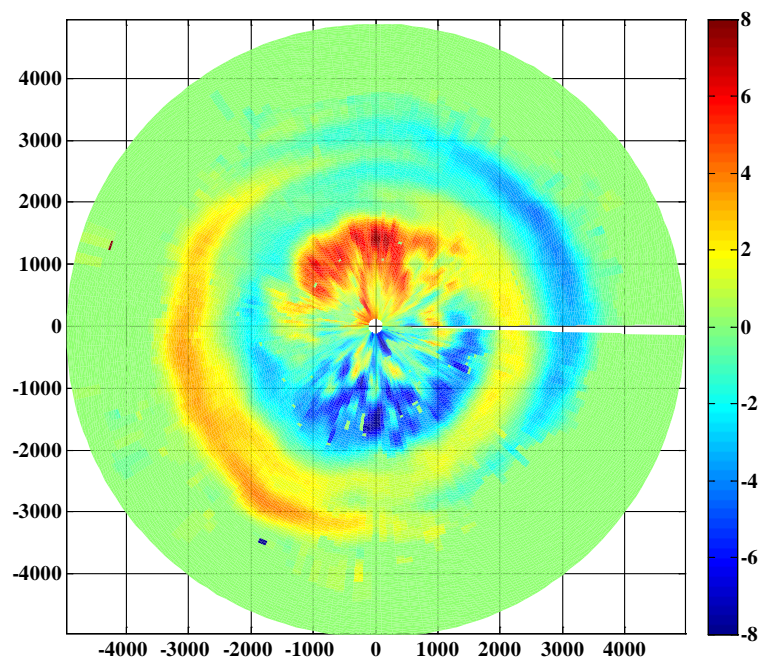

Fig. 8. Wind shear calculation results for PPI mode 


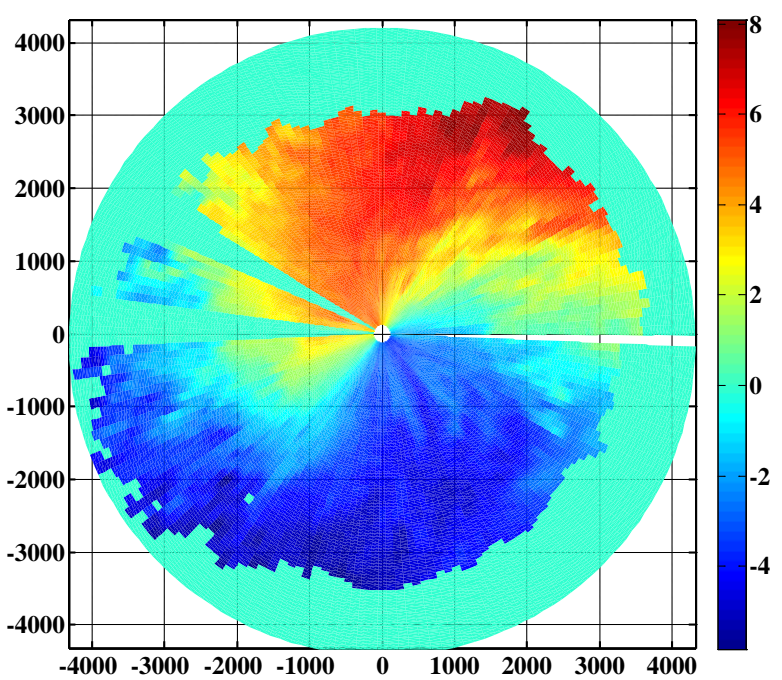

Fig. 9. Wind field in PPI mode in wind shear conditions due to change in wind direction

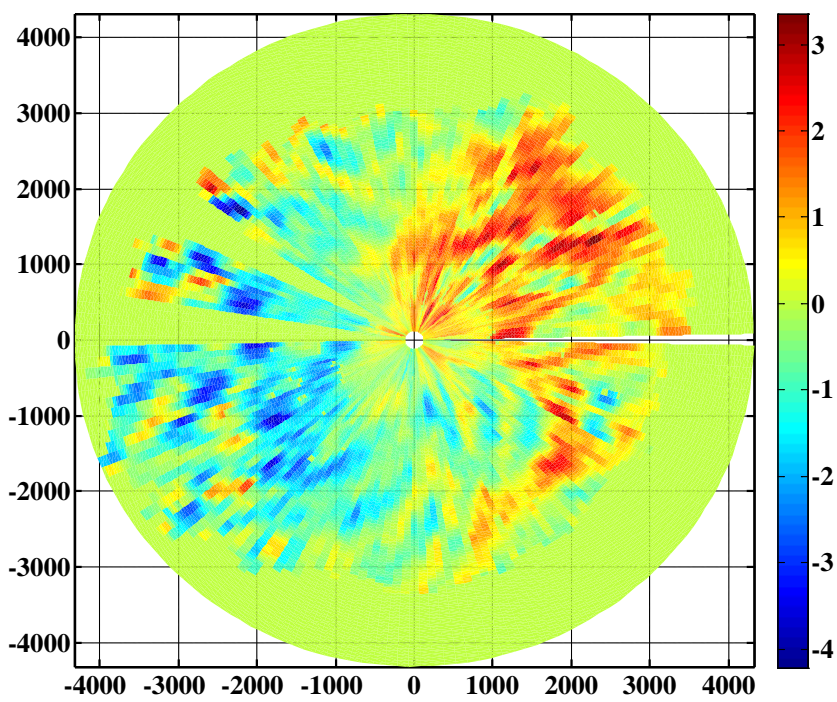

Fig. 10. Wind shear field due to change in wind direction

In conclusion, we will demonstrate the possibility of applying the algorithms presented herewith for the analysis of wind shear phenomena when performing measurements with a Doppler lidar in RHI (Range Height Indicator) mode. RHI mode corresponds to measurements made in a certain range of elevation angles for a fixed azimuthal direction.

Figure 11 shows the radial velocity field measured in RHI mode. The presented measurement data have a characteristic feature related to the presence of clouds in the height range of 2000-3000m. The presence of clouds is illustrated in Figure 12 which shows the distribution of the signal-to-noise ratio (SNR) values that reflects the intensity of the backscatter signal which increases significantly while passing the boundary of the cloud layer and weakens inside the cloud layer.

The qualitative picture of wind speed changing with hight is characterized by the fact that in the height range of $600-2000 \mathrm{~m}$ there is a wind shear in the sub-cloud layer due to intense convective movement of air masses., The value of the wind speed radial component decreases inside the clouds which is most likely due to a change in the wind speed direction that leads to a decrease in the wind speed projection on the scanning direction. Above the cloud layer (higher than $3000 \mathrm{~m}$ ) the radial component of the wind speed increases again.

This qualitative analysis is confirmed by the calculation results for the wind shear parameters presented in Figure 13. In this figure, the boundaries of intense changes in the radial wind speed are clearly visible: the negative wind shear at $500 \mathrm{~m}$, the positive wind shear at $2000 \mathrm{~m}$ and again the negative wind shear at $3000 \mathrm{~m}$.

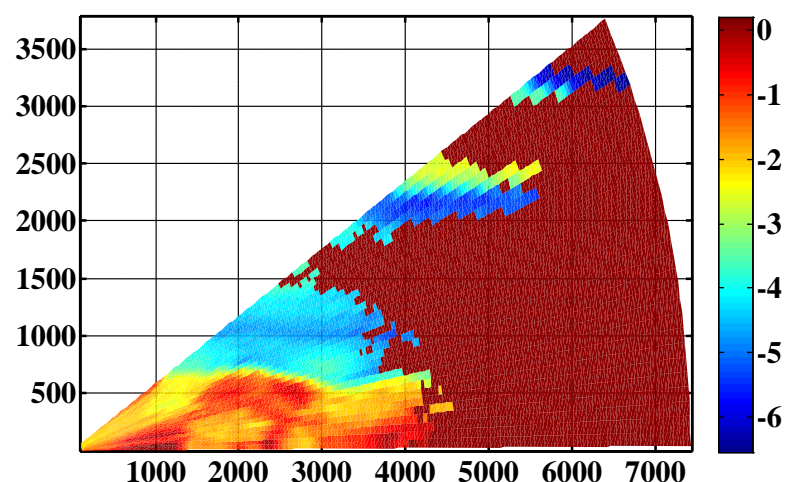

Fig. 11. Wind radial velocity field in RHI mode.

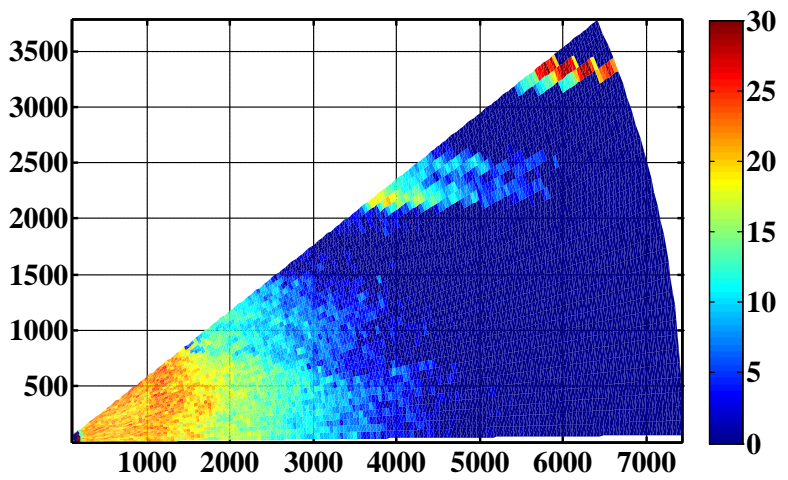

Fig. 12. SNR field in RHI mode.

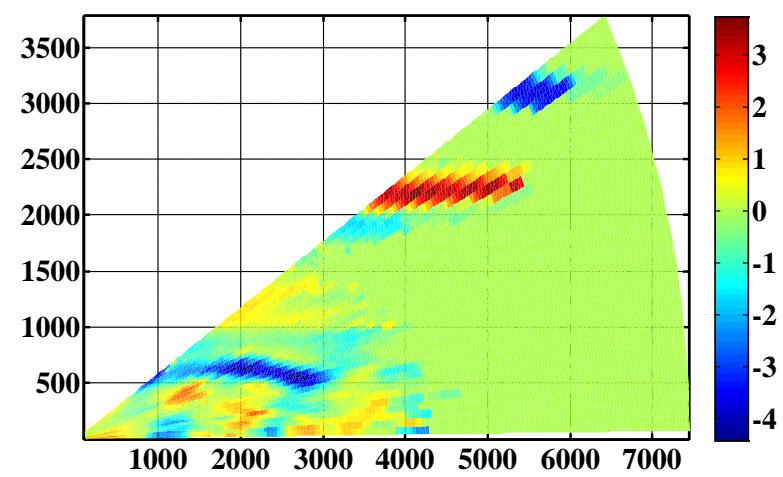

Fig. 13. Windshear field in RHI mode

\section{Conclusion}

The present paper reviews the issues of implementing the algorithms for calculating the parameters of hazardous wind phenomena in the PPI scanning mode of the Doppler pulsed lidar. The analyzed algorithms are based on the construction of approximating dependences 
of changes in the wind characteristics along a given direction at a distance determining wind shear parameters. Concurrently, additional multi-step procedures for analyzing the reliability of measurement data are applied, taking into account both the quality of the received signal (signal-to-noise ratio) and outliers in the measurement data. In addition, smoothing algorithms are applied before pre-processing procedures for calculating the wind shear parameters to partially solve the problem of recovering the missing measurement data.

The functionality of the algorithms is demonstrated on a number of examples of data processing of lidar scanning using WINDEX-5000 pulse Doppler lidar.

The application of the presented algorithms ensures both qualitative assessment of the wind shear presence based on the results of visual analysis of the nature of radial velocities distribution and obtaining quantitative estimates of the intensity of wind shear phenomena, as well as their spatial distribution.

The work was partially supported by the Russian Foundation for Basic Research (project No. 16-07-01072).

\section{References}

1. K. K. Hon, P. W. Chan, Y. Y. Chiu, Wenbo Tang, Advances in Meteorology, vol. 2014 (2014) 537.

2. C. M. Shun and P.W.Chan, Journal of Atmospheric and Oceanic Technology, vol. 25, no. 5, 2008, pp. 637-655.

3. H.A.Kafiabad, P.W.Chan, G.Haller, Journal of Atmospheric and Oceanic Technology, vol. 30, no. 12, 2013, pp. 2808-2819.

4. P. W. Chan, Y. F. Lee, J. of Atmospheric and Oceanic Technology, vol. 29, no. 2, pp. 207-220, 2012.

5. A. Weipert, S. Kauczok, R. Hannesen, T. Ernsdorf, B Stiller, ERAD 2014 Abstract ID 058.

6. P.W. Chan, C M Shun, K C Wu, Reprint 613, 2006.

7. P.W. Chan, C.M. Shun \& M.L. Kuo, Reprint 871, 2010.

8. Manual on Low-Level Wind Shear, ICAO Doc 9817, 2005.

9. ISO 28902-2:2017.

10. P.W.Chan, M.L.Kuo, Proc. 24th International Laser Radar Conference, 23-27 June 2008, Boulder, Colorado, USA.

11. P.W.Chan, Weather, vol. 67, issue 7, 2012, pp. 176181.

12. P.W.Chan, Meteorologische Zeitschrift Vol. 21 No. 2 (2012), p. $193-204$.

13. R. A. Brown, V. T. Wood, A guide for interpreting doppler velocity patterns: Northern Hemisphere Edition, 2007. 